\title{
Evaluating Computational Predictions of the Relative Stabilities of Polymorphic Pharmaceuticals
}

\author{
KATIE R. MITCHELL-KOCH, ADAM J. MATZGER \\ Department of Chemistry, Macromolecular Science and Engineering Program, University of Michigan, \\ Ann Arbor, Michigan 48109-1055
}

Received 21 April 2007; revised 1 June 2007; accepted 12 June 2007

Published online in Wiley InterScience (www.interscience.wiley.com). DOI 10.1002/jps.21127

\begin{abstract}
The ability of computational methods to describe the relative energies of polymorphic pharmaceuticals is investigated for a diverse array of compounds. The initial molecular geometries were taken from crystal structures, and energy differences between polymorphic pairs were calculated with various geometry optimization methods. Results using molecular mechanics were compared to experimental calorimetric data and periodic density functional theory (DFT) calculations. The best agreement with experimental heats of transition was shown with energies calculated from geometry optimizations using the Compass force field. Calculations that optimized atomic positions with the Compass force field gave correct energy rankings for all 11 polymorphic pairs studied, with an average deviation of $0.61 \mathrm{kcal} / \mathrm{mol}$ from experimental results. These findings suggest that computational methods are poised to predict enthalpy differences between polymorphic forms with levels of accuracy that are quite acceptable when proper approaches are employed. (c) 2007 Wiley-Liss, Inc. and the American Pharmacists Association J Pharm Sci 97:2121-2129, 2008
\end{abstract}

Keywords: computer modeling; molecular modeling; DFT; force field; thermodynamics; polymorphism; crystal; stability

\section{INTRODUCTION}

An important prerequisite for crystal structure prediction is the ability of a computational method to correctly rank the relative energies of different polymorphic packing structures. The correct energy ranking of polymorphs is also important to describe hypothetical or newly discovered polymorphic systems, which are sometimes energetically characterized by computational methods in lieu of calorimetric data. While many computa-

Tables with calculated energies, calculated transition energies, and experimental heats of transition. This material is available free of charge via the Internet (http://www. interscience.wiley.com/).

Katie R. Mitchell-Koch's present address is Department of Chemistry, University of Kansas, 1251 Wescoe Hall Drive, 2010 Malott Hall, Lawrence, Kansas 66045.

Correspondence to: Adam J. Matzger (Telephone: 1-734615-6627; Fax: 1-734-615-8553; E-mail: matzger@umich.edu)

Journal of Pharmaceutical Sciences, Vol. 97, 2121-2129 (2008)

(c) 2007 Wiley-Liss, Inc. and the American Pharmacists Association tional methods have been validated through comparisons with structural data, ${ }^{1}$ fewer have been evaluated for their ability to reproduce experimental energies. ${ }^{2}$ Most work comparing experimental and computational energy differences of polymorphic compounds has been done for isolated systems, ${ }^{3}$ and there is a dearth of energetic characterizations across classes of materials. ${ }^{4}$ We have undertaken a systematic study to evaluate the ability of computational methods to predict the relative energies ${ }^{5}$ for a prominent class of materials: polymorphic pharmaceuticals. Crystal polymorphism ${ }^{6}$ is a critical issue in pharmaceutical manufacture and dosage, because diverse properties, including solubility and stability, depend on crystal form. ${ }^{7}$

In this study, a number of computational methods were used to calculate energy differences between pairs of crystal polymorphs, and the values were compared with experimentally determined heats of transition $\left(\Delta H_{\mathrm{trs}}\right)$. By using a 
variety of organic pharmaceutical compounds with diverse functional groups, this systematic investigation sought to determine if molecular mechanics methods are able to accurately rank the relative energies of arbitrary polymorphs, and if so, how well they reproduce experimental energy differences. These methods are a particularly attractive approach because of their low computational cost and ability to rapidly carry out calculations on large molecules and periodic systems. In addition, because they rely on empirical potentials for intermolecular interactions, they do not suffer from the problems in describing dispersive interactions that $a b$ initio methods without explicit treatment of correlation encounter. Such problems can be partially addressed by density functional theory (DFT), with the substantial advantage over mechanics methods that extensive parameterization for new functionality is not needed. The molecular mechanics calculations were compared to these much more computationally intensive DFT calculations for several of the systems examined.

\section{METHODS}

The data set was chosen to contain pharmaceuticals with high quality structural and calorimetric characterization, such that direct comparison could be made between experimental and computational results. If multiple crystal structures were available for a given pharmaceutical, structures acquired at low temperature or with lower $R$-values were selected. Specific compounds were chosen to create a set of pharmaceuticals with a variety of functional groups and hydrogen bonding motifs. Although the choice of systems is necessarily somewhat arbitrary, all molecules examined are included in the results presented without regard to agreement between experimental and computational results. Figure 1 shows the chemical structures of the molecules studied, along with their Cambridge structural database (CSD) reference codes.

Crystal structures were obtained from the CSD, and correct bond orders were assigned in the model using the crystal builder module in Cerius ${ }^{2}$. The force field was loaded, and force field typing was applied to all of the atoms in the structure. Two force fields were used for this study: Dreiding $2.21^{8}$ and Compass. ${ }^{9}$ Dreiding is a generic force field designed for use with organic, biological, and main group inorganic compounds in vacuo or in periodic systems. Compass is a condensed-phase optimized, $a b$ initio force field designed for use with organic and inorganic molecules and materials.

For calculations conducted with the Dreiding 2.21 force field, the charges were calculated with<smiles>COc1ccc2c(c1)c(CC(=O)O)c(C)n2C(=O)c1ccc(Cl)cc1</smiles>

indomethacin form $\alpha$ INDMET02 form $\gamma$ INDMET03<smiles>Cc1cc(NS(=O)(=O)c2ccc(N)cc2)no1</smiles>

sulfamethoxazole form I SLFNMB01 form II SLFNMB02<smiles>CC(=O)Nc1ccc(O)cc1</smiles>

acetaminophen form I HXACAN07 form II HXACAN08

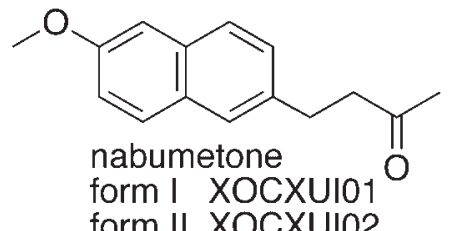<smiles>Nc1ccc(S(=O)(=O)Nc2nccs2)cc1</smiles>

sulfathiazole form I SUTHAZ01 form II SUTHAZ

Figure 1. Pharmaceutical compounds studied: polymorphic forms and CSD codes. 
the charge equilibration method, ${ }^{10}$ which was shown to give reasonable results for geometry optimizations in a wide survey of organic crystals. ${ }^{11}$ In the case of the Compass force field, the atomic partial charges are calculated automatically with the atom typing function. For several of the compounds studied, the Compass force field as implemented in Cerius ${ }^{2}$ version 4.6 was not parameterized for the functional groups present. Hence, calculations for carbamazepine, sulfathiazole, and sulfamethoxazole used the Compass 98-01 force field, which was suitably parameterized.

The default parameters in Cerius ${ }^{2}$ for longrange and nonbonding interactions, which vary by force field, were used for all of the results herein. It was found that changing the methods for calculating van der Waals and Coulombic interactions usually did not change the relative energy ordering, but did lead to greater overestimates of the energy differences between polymorphs.

The calculated energies were divided by the number of molecules per unit cell such that energies are given in kcal per mole of molecules rather than kcal per mole of unit cells. In this way, direct comparison could be made between calculated energies and experimental results. The energies of transition $\left(\Delta E_{\mathrm{trs}}\right)$ were calculated by finding the difference in calculated energies between two polymorphs (Eq. 1).

$$
\Delta E_{\mathrm{trs}}(\text { form II } \rightarrow \text { I })=E_{\text {form I }}-E_{\text {form II }}
$$

It is a convention in many sets of calorimetric data to give enthalpically favored heats of transition $\left(\Delta H_{\text {trs }}\right)$ as a positive number. In this article, all heats of transition follow the convention of Eq. (1); thus, all exothermic transitions have a negative sign.

\section{RESULTS AND DISCUSSION}

A number of computational approaches were explored for the set of polymorphs. The simplest calculation that can be employed is a single point energy, in which the energy for a crystal structure is directly calculated. Other strategies include constrained geometry optimizations, in which some atomic positions are allowed to vary, and full geometry optimizations with all atomic positions and cell parameters unconstrained. Figure 2 presents the geometry optimizations used in this study. For each type of calculation, the energy differences between pairs of polymorphs

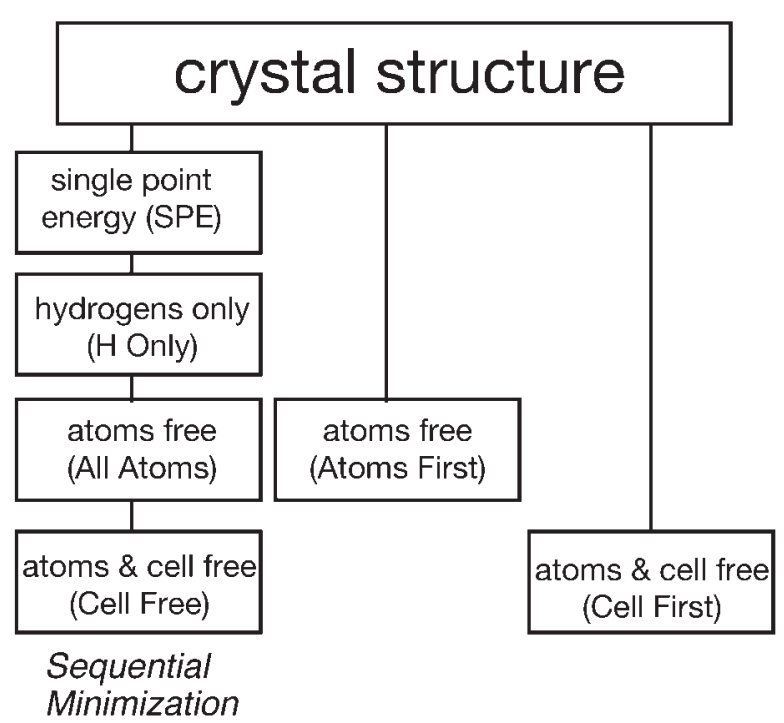

Figure 2. Calculations and geometry optimization sequences.

were calculated. The various calculation methods are discussed below. The results are summarized in Figures 3 and 4, which give the average deviation of calculated results from experimental data and the frequency of correct energy ordering predicted by each method.

\section{SPE}

The most straightforward route to calculate energy differences between polymorphs is to employ geometries directly from the crystal structure: a single-point energy calculation ("SPE"). However, X-ray diffraction systematically underestimates bond lengths involving hydrogen atoms due to the displacement of the electron density from the nuclear position; therefore, it cannot provide reasonable geometries for computation. Moreover, these bonds are subject to large uncertainties, such that the hydrogen positions are not systematically shortened, and these contracted bonds containing hydrogen lead to a dramatic overestimation of the calculated energies. Due to the magnitude of the covalent bond energies, slight differences in bond lengths in the set of polymorphs overshadow differences in noncovalent interactions. Thus, single point energy calculations are not a valid means of characterizing the energetics of polymorphs. The shortcomings of the calculation were reflected in the results: for both force fields, calculated $\Delta E_{\text {trs }}$ were in poor agreement with experimental values, and the correct 


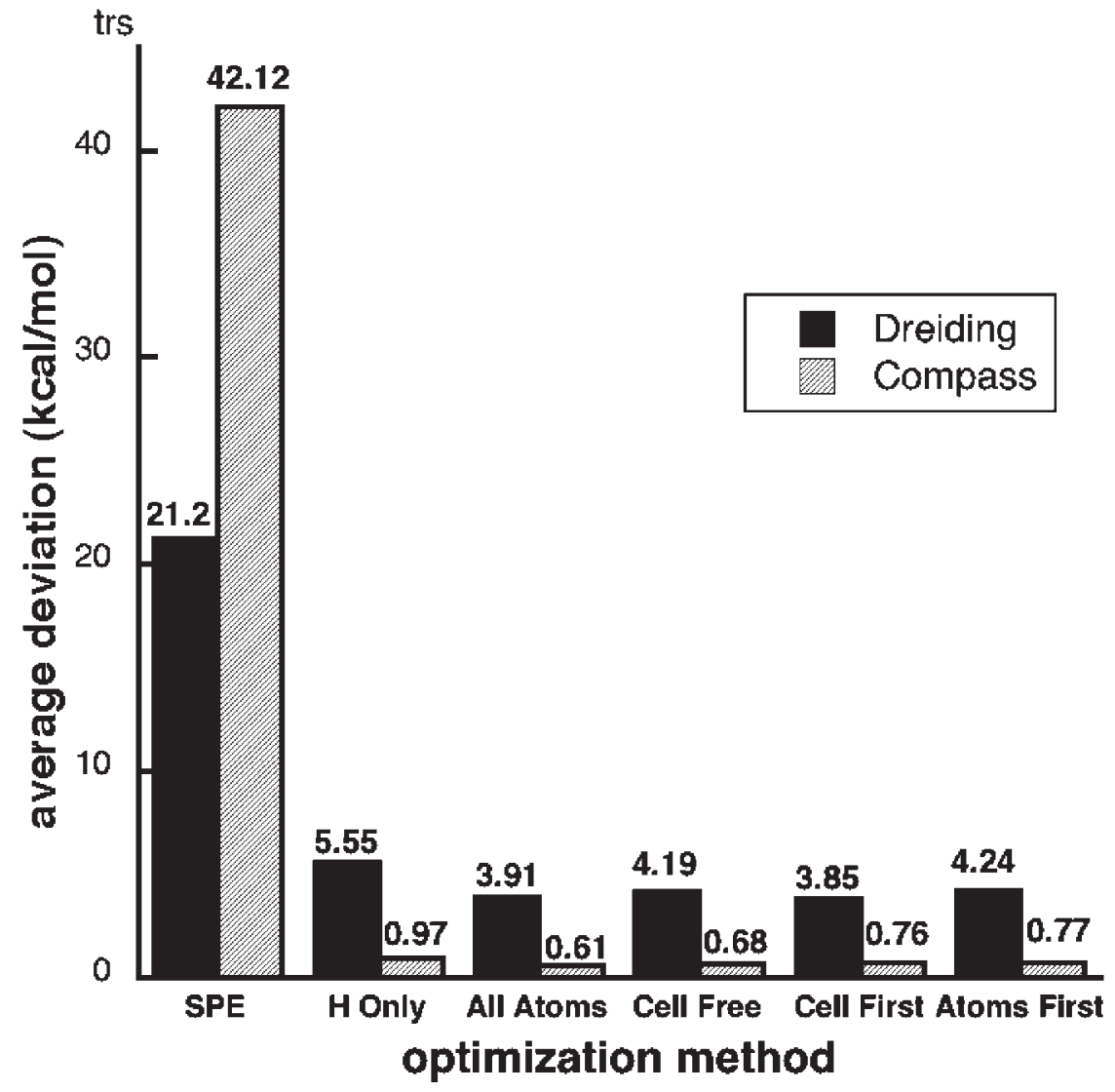

Figure 3. Average deviation from experimental $\Delta H_{\text {trs }}$ for each optimization method and force field.

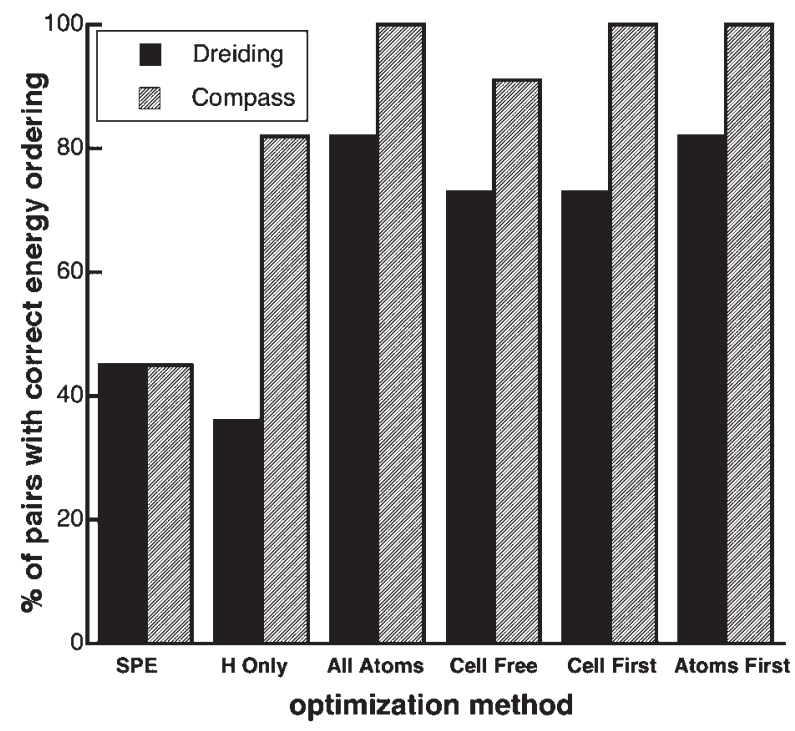

Figure 4. Percentage of polymorphic pairs with correctly predicted relative stabilities. energy ordering was predicted for only 5 of the 11 polymorphic pairs.

\section{H Only}

To ameliorate the shortcomings of single point energy calculations, while retaining the more reliably determined experimental results, a constrained geometry minimization ("H Only") was carried out in which the positions of the hydrogen atoms were optimized. All other atomic positions and cell parameters were constrained. By adjusting the positions of the hydrogen atoms, the energies of the systems were lowered dramatically: with the Compass force field, the average energy difference between $S P E$ calculations and $H$ Only minimizations was $122 \mathrm{kcal} / \mathrm{mol}$; for the Dreiding force field, the average energy difference was $49.9 \mathrm{kcal} / \mathrm{mol}$. The dramatic lowering of energy, due to correcting the unphysical short 
crystallographic $\mathrm{C}-\mathrm{H}, \mathrm{N}-\mathrm{H}$, and $\mathrm{O}-\mathrm{H}$ distances, should provide more reliable energy values for the systems, while still maintaining fidelity to the original crystal structures. Indeed, this was true for the Compass force field: this method showed considerable improvement over the SPE calculations in the ability of the method to predict the correct energy ordering of polymorphic pairs, with 9 of 11 matching experimental ordering, and an average deviation from the experimental $\Delta H_{\text {trs }}$ value of $0.97 \mathrm{kcal} / \mathrm{mol}$. However, for the Dreiding force field, the method gave poor agreement with the experimental energy ordering (4 of 11 correctly predicted) and an average deviation of $5.55 \mathrm{kcal} / \mathrm{mol}$ from experimental differences in heats of fusion.

\section{All Atoms}

Calculations were carried out in which all atomic positions were allowed to vary, while the unit cell lengths and angles were held constant. The high sensitivity of fixed geometry calculations to perturbations in bond length due to experimental errors was demonstrated in a study of lattice energy minimizations of multiple paracetamol (acetaminophen) crystal structures by Beyer and Price. ${ }^{12}$ Uncertainties in the crystallographically determined molecular structure can lead to errors in covalent bond energies that overwhelm differences in lattice energy terms, leading to inaccurate energy differences in a set of polymorphs. For this reason, calculations in which the atomic positions are allowed to relax to their lowest energy position within the lattice may offer an improvement in accuracy over $H$ Only optimizations. Indeed, this was seen for both force fields. ${ }^{13}$ For Compass force field, the average difference between $H$ Only and All Atoms energies was $9.5 \mathrm{kcal} / \mathrm{mol}$; the differences between $H$ Only and All Atoms calculations were larger for Dreiding force field ( $34.4 \mathrm{kcal} / \mathrm{mol}$ average). The energy differences between the $H$ Only and All Atoms minimizations are fairly large, and these energy changes and the discrepancies between the two force fields most likely correspond to the adjustment of the heavy atom bond lengths from the crystallographically determined positions to the equilibrium bond lengths for the given force fields.

For each force field, the All Atoms calculations gave the best agreement with experimental results within this survey: for the Compass force field, correct energy ordering was predicted for all
11 pairs, with an average deviation of $0.61 \mathrm{kcal} / \mathrm{mol}$ from experimental $\Delta H_{\text {trs }}$; the Dreiding force field gave correct energy ordering for 9 of 11 pairs, with an average deviation of $3.91 \mathrm{kcal} / \mathrm{mol}$.

\section{Cell Free}

If the intermolecular energy terms in a particular model are, on balance, too repulsive, there will be a stability bias toward the least dense crystal. Moreover, the temperature at which crystal structures are obtained may vary among polymorphs, and lower temperatures cause an anisotropic contraction in cell volume. In order to eliminate any bias based on density, a minimization was carried out in which the cell parameters (lengths and angles) were allowed to vary along with all atomic coordinates ("Cell Free").

When the Cell Free calculations were carried out, they were monitored for changes in the cell angles. A nearly isotropic expansion of cell volume was often observed during these calculations, along with slight $(<5 \%)$ changes in cell angles. Notable exceptions are sulfamethoxazole and sulfathiazole, for which drastic changes in the $\beta$ angle were observed when cell parameters were allowed to vary. The largest changes were seen in the sulfathiazole Cell Free calculations with Compass force field, with a change of $18^{\circ}$ in the $\beta$ angle of form II; indeed, this led to incorrect energy ordering. More importantly, this illustrates a caveat of the full geometry optimizations: when atomic positions and cell parameters are allowed to vary, polymorphic transitions can be predicted. In such cases, the "optimized structure" and calculated energy no longer represents the starting crystallographic structure. ${ }^{14}$ Cell Free optimizations must be monitored closely for such geometric changes lest the comparisons between forms become invalid. In spite of these drawbacks, calculated results had reasonable agreement with experimental data, but less so than the All Atoms minimizations. The Compass force field gave correct energy ordering for 10 of 11 pairs, with $0.68 \mathrm{kcal} / \mathrm{mol}$ average deviation from $\Delta H_{\text {trs }}$; the Dreiding force field gave correct energy ordering for 8 of 11 pairs with an average deviation of $4.19 \mathrm{kcal} / \mathrm{mol}$.

\section{Order of Optimization}

The order in which the above calculations were carried out was varied, in order to determine the 
effect that the starting point on the potential energy surface has on the resulting minimized geometry and energy in the All Atoms and Cell Free minimizations. In the calculations in which a full atomic geometry optimization was carried out without a prior adjustment of the bonds containing hydrogen ("Atoms First," see Fig. 1), the energy ordering matched the accuracy of the All Atoms minimization. The average deviations for both force fields were also comparable, although for both force fields, the All Atoms minimization gave slightly better agreement with experimental $\Delta H_{\text {trs }}$ values. Within the Compass force field, the calculated energies for the Atoms First minimization matched the calculated energies with the All Atoms minimization for 13 out of 14 structures investigated. For the same two minimizations with Dreiding force field, the calculated energies were identical for only half of the polymorphic pairs studied. The optimized structures obtained with Dreiding force field are more dependent on the minimization sequence used.

\section{Cell First}

In the "Cell First" geometry optimization, the atomic coordinates and cell parameters were unconstrained, and the energy was minimized starting directly from the crystal structure. For the Compass force field, the average energy difference between the single point energy (the energy of the starting crystal structure) and the Cell First minimization was $132 \mathrm{kcal} / \mathrm{mol}$. In contrast, the average energy difference between the All Atoms and Cell Free minimizations is $0.97 \mathrm{kcal} / \mathrm{mol}$. In spite of the vastly different starting points on the potential energy surface, the Cell Free and Cell First minimizations only led to different optimized structures and energies being calculated for 2 of the 14 polymorphs.

For the Dreiding force field, the average energy difference between the starting structure and the Cell First optimized structure was $88.8 \mathrm{kcal} / \mathrm{mol}$. The average energy difference between the All Atoms and Cell Free minimizations was $4.7 \mathrm{kcal} / \mathrm{mol}$. Compared to the Compass force field, energy differences vary more among each type of optimization with the Dreiding force field. The Cell First energies matched the Cell Free energies for only 2 of the 14 structures studied (both forms of nabumetone), which is reflective of the large structural and energetic differences in the starting points for each calculation. This again demonstrates that, especially for the Dreiding force field, the sequence in which geometry optimizations are carried out can influence the results obtained.

\section{Density Functional Theory Calculations}

In crystal structure prediction methodology, structures that lie within $2.4 \mathrm{kcal} / \mathrm{mol}(10 \mathrm{~kJ} / \mathrm{mol})$ of the global minimum are often considered to be viable structures. ${ }^{15}$ Although all polymorphic pairs had experimental energy differences of less than $1.63 \mathrm{kcal} / \mathrm{mol}$, some of the methods used in this study routinely calculated energy differences higher than $2.4 \mathrm{kcal} / \mathrm{mol}$. This was the case for the single point energies with both force fields, and many calculations using different geometry optimization strategies with the Dreiding force field. Because energy rankings are crucial in crystal structure prediction, highly accurate force fields as well as robust geometry minimization procedures are critical to the development of this field. ${ }^{16}$

An alternative method that has been used to describe the energies of periodic systems is DFT calculations using a plane wave basis set. These methods have great utility when dramatic structural changes occur, as in the case of extended solids, ${ }^{17}$ and do not suffer from the need to parameterize extensively. We sought to compare molecular mechanics results with DFT calculations. Due to the computational expense, the systems and methods employed in this DFT study were limited. With large periodic systems, full geometry optimizations with DFT are computationally intensive and not routine; therefore, constrained optimizations that allowed the hydrogen positions to vary were implemented. The crystal structures were preoptimized with an H Only minimization using molecular mechanics in Cerius ${ }^{2}{ }^{18}$ The DFT calculations were carried out with the CASTEP code, ${ }^{19}$ using GGA-PW91 theory ${ }^{20}$ and a fine basis set. Polymorphs of acetaminophen, nabumetone, indomethacin, sulfathiazole, and sulfamethoxazole were studied. The results are given in Table $1 .{ }^{21}$ In all cases but nabumetone, the transition energies were overestimated by $H$ Only DFT calculations with the PW91 functional. Within this limited investigation, DFT calculations were found to be neither an efficient nor reliable method, relative to molecular mechanics methods, for calculating the relative energies of polymorphs. 
Table 1. Summary of $\Delta E_{\text {trs }}$ from $H$ only DFT Calculations and Comparison with Experimental Values

\begin{tabular}{lcccr}
\hline Compound & Transition & $\begin{array}{c}\text { Calculated } \\
\Delta E_{\text {trs }}(\mathrm{kcal} / \mathrm{mol})\end{array}$ & $\begin{array}{c}\text { Experimental } \\
\Delta H_{\text {trs }}{ }^{21}(\mathrm{kcal} / \mathrm{mol})\end{array}$ & $\begin{array}{r}\text { Deviation } \\
(\mathrm{kcal} / \mathrm{mol})\end{array}$ \\
\hline Acetaminophen & $\mathrm{II} \rightarrow \mathrm{I}$ & -1.71 & $-0.78^{21 \mathrm{a}}$ & 0.93 \\
Indomethacin & $\alpha \rightarrow \gamma$ & -2.44 & $-0.27^{21 \mathrm{~b}}$ & \\
Nabumetone & $\mathrm{II} \rightarrow \mathrm{I}$ & -1.54 & $-1.63^{21 \mathrm{c}}$ & \\
Sulfamethoxazole & $\mathrm{II} \rightarrow \mathrm{I}$ & 4.31 & $-0.34^{21 \mathrm{~d}}$ & \\
Sulfathiazole & $\mathrm{II} \rightarrow \mathrm{I}$ & -13.19 & $-1.62^{21 \mathrm{~d}}$ & \\
\hline
\end{tabular}

\section{CONCLUSIONS}

Because of the low computational expense of molecular mechanics methods and good agreement with experimental results using the Compass force field, such methods for geometry minimizations of known crystal structures have been shown to be the best computational approach to describe the energetics of polymorphic pharmaceutical compounds in this survey. Table 2 summarizes how well each method agrees with experimental data. Quantitative agreement was rarely seen with Dreiding force field calculations, at best giving agreement within $1 \mathrm{kcal} / \mathrm{mol}$ for 4 of the 11 pairs in the All Atoms and Atoms First minimizations. More promising, the All Atoms and Cell Free minimizations using the Compass force field agreed with experimental results within $1 \mathrm{kcal} / \mathrm{mol}$ for 9 of the 11 pairs. It is encouraging that some methods are able to consistently predict the correct energy rankings, as observed with all minimization methods using the Compass force field.

DFT methods are less versatile: fewer types of calculations can be attempted on organic crystals because of computational expense. This brief survey of DFT calculations indicates that the $H$ Only minimization with the PW91 functional does not, in general, give reliable values for heats of transition. Indeed, the calculations largely overestimated the $\Delta H_{\text {trs }}$ for three of the five pairs studied. However, because four of the five energy rankings were correct with DFT methods, this approach may be useful as a method to corroborate energy rankings found by molecular mechanics calculations. In conclusion, the best method for calculating relative energies in this survey of pharmaceutical polymorphs was a geometry optimization of all atomic coordinates with the unit cell parameters constrained using the Compass force field.

Table 2. Summary of all Computational Methods and Their Agreement with Experimental $\Delta H_{\text {trs }}$

\begin{tabular}{lcccc}
\hline & $\begin{array}{c}\text { Number Pairs With } \\
\text { Correct Energy } \\
\text { Ordering }\end{array}$ & $\begin{array}{c}\text { Average Deviation From } \\
\text { Experimental } \\
\Delta H_{\text {trs }}\end{array}$ & $\begin{array}{c}\text { Standard Deviation } \\
\text { From Experimental } \\
\Delta H_{\text {trs }}\end{array}$ & $\begin{array}{c}\text { Number Pairs With } \\
<1.0 \text { kcal/mol } \\
\text { Deviation }\end{array}$ \\
\hline Compass FF & & & & \\
SPE & 5 & 42.12 & 59.74 & 1 \\
H Only & 9 & 0.97 & 1.33 & 9 \\
All atoms & 11 & 0.61 & 0.55 & 9 \\
Cell Free & 10 & 0.68 & 0.92 & 8 \\
Cell First & 11 & 0.76 & 0.70 & 7 \\
Atoms First & 11 & 0.77 & 0.64 & 0 \\
Dreiding FF & & & & 3 \\
SPE & 5 & 21.2 & 8.15 & 2 \\
H Only & 4 & 5.55 & 6.38 & 1 \\
All Atoms & 9 & 3.91 & 5.40 & 4 \\
Cell Free & 8 & 4.19 & 5.39 & 2 \\
Cell First & 8 & 3.85 & 6.77 & \\
Atoms First & 9 & 4.24 & 4.67 & 2 \\
DFT (PW91) & & & & \\
H Only & 4 of 5 & 3.88 & & \\
\hline
\end{tabular}




\section{ACKNOWLEDGMENTS}

This work was supported by the National Science Foundation under grant CHE-0316250. K. M. K. gratefully acknowledges the support by the Fannie and John Hertz Foundation.

\section{REFERENCES}

1. For recent examples of crystal structure prediction methods compared with known crystallographic data see: (a) Lommerse JPM, Motherwell WDS, Ammon HL, Dunitz JD, Gavezzotti A, Hofmann DWM, Leusen FJJ, Mooij WTM, Price SL, Schweizer B, Schmidt MU, van Eijck BP, Verwer P, Williams DE, 2000. A test of crystal structure predictions of small organic molecules. Acta Crystallogr B56:697-714; (b) Motherwell WDS, Ammon HL, Dunitz JD, Dzyabchenko A, Erk P, Gavezzotti A, Hofmann DWM, Leusen FJJ, Lommerse JPM, Mooij WTM, Price SL, Scheraga H, Schweizer B, Schmidt MU, van Eijck BP, Verwer P, Williams DE, 2002. Crystal structure prediction of small organic molecules: A second blind test. Acta Crystallogr B58:647-661; (c) Day GM, Motherwell WDS, Ammon HL, Boerrigter SXM, Della Valle RG, Venuti E, Dzyabchenko A, Dunitz JD, Schweizer B, van Eijck BP, Erk P, Facelli JC, Bazterra VE, Ferraro MB, Hofmann DWM, Leusen FJJ, Liang C, Pantelides CC, Karamertzanis PG, Price SL, Lewis TC, Nowell H, Torrisi A, Scheraga HA, Arnautova YA, Schmidt MU, Verwer P, 2005. A third blind test of crystal structure prediction. Acta Crystallogr B61:511-527; (d) Day GM, Chisholm J, Shan N, Motherwell WDS, Jones W, 2004. Assessment of lattice energy minimization for the prediction of molecular organic crystal structures. Cryst Growth Des 4:1327-1340; (e) Raiteri P, Martoňák R, Parrinello M, 2005. Exploring polymorphism: The case of benzene. Angew Chem Int Ed 44:3769-3773; (f) Lewis TC, Tocher DA, Price SL, 2004. An experimental and theoretical search for polymorphs of barbituric acid: The challenges of even limited conformational flexibility. Cryst Growth Des 4:979-987; (g) Karamertzanis PG, Pantelides CC, 2005. Ab initio crystal structure prediction-I. Rigid molecules. J Comput Chem 26:304-324; (h) Jagielska A, Arnautova YA, Scheraga HA, 2004. Derivation of a new force field for crystalstructure prediction using global optimization: Nonbonded potential parameters for amines, imidazoles, amides, and carboxylic acids. J Phys Chem B 108: 12181-12196.

2. Recent work has compared computational lattice energies and experimental heats of sublimation: (a) Feng S, Li T, 2006. Predicting lattice energy of organic crystals by density functional theory with empirically corrected dispersion energy. J Chem Theory Comput 2:149-156; (b) Gavezzotti A, 2005. Quantitative ranking of crystal packing modes by systematic calculations on potential energies and vibrational amplitudes of molecular dimers. J Chem Theory Comput 1:834-840.

3. (a) Hulme AT, Price SL, Tocher DA, 2005. A new polymorph of 5-fluorouracil found following computational crystal structure predictions. J Am Chem Soc 127:1116-1117; (b) van Eijck BP, 2001. Ab initio crystal structure predictions for flexible hydrogen-bonded molecules. Part III. Effect of lattice vibrations. J Comput Chem 22:816826.

4. A survey carried out by Gavezzotti and Filipini involved the comparison of experimental heats of transition, calculated enthalpy differences for a set of organic polymorphic compounds. The calculations only calculated intermolecular forces, thus, it could not evaluate differences in conformational polymorphs. Gavezzotti A, Filipini G, 1995. Polymorphic forms of organic crystals at room conditions: Thermodynamic and structural implications. J Am Chem Soc 117:12299-12305.

5. For a discussion of the relationships between enthalpy $(\Delta \mathrm{H})$ and stability, which is determined by the free energy $(\Delta G)$, see, for example, (a) Yu L, 1995. Inferring thermodynamic stability relationship of polymorphs from melting data. J Pharm Sci 84:966-974.5.;

(b) Burger A, Ramberger R, 1979. On the polymorphism of pharmaceuticals and other molecular crystals. I. Theory of thermodynamic rules. Microchim Acta 72:259-271.

6. Rodríguez-Spong B, Price CP, Jayasankar A, Matzger AJ, Rodríguez-Hornedo N. 2004. General principles of pharmaceutical solid polymorphism: A supramolecular perspective. Adv Drug Deliv Rev 56:241-274.

7. Brittain HG, Grant DJW. 1999. Effects of polymorphism and solid-state solvation on solubility and dissolution rate. In: Brittain HG, editor. Polymorphism in pharmaceutical solids. New York: Marcel Dekker, Inc. pp 279-330.

8. Mayo SL, Olafson BD, Goddard WA. 1990. Dreiding-a generic force-field for molecular simulations. J Phys Chem 94:8897-8909.

9. Sun H. 1998. COMPASS: An ab initio force-field optimized for condensed-phase applications-overview with details on alkane and benzene compounds. J Phys Chem B 102:7338-7364.

10. Rappé AK, Goddard WA. 1991. Charge equilibration for molecular-dynamics simulations. J Phys Chem 95:3358-3363.

11. Brodersen S, Wilke S, Leusen FJJ, Engel GE. 2005. Comparison of static and fluctuating charge models for force-field methods applied to organic crystals. Cryst Growth Des 5:925-933. 
12. Beyer T, Price SL. 2000. The errors in lattice energy minimisation studies: Sensitivity to experimental variations in the molecular structure of paracetamol. CrystEngComm 2:183-190.

13. With the All Atoms calculation, it is possible that polymorphic transitions can be predicted by changes in covalent bond lengths, torsion angles, or hydrogen bonding patterns. When carrying out these calculations, the system can be monitored for changes in torsion angles and hydrogen bond motifs. However, among experimental polymorphs, differences in atomic position are usually accompanied by differences in unit cell parameters and symmetry. Because the cell lengths and angles are constrained in these calculations, polymorphic transitions between different space groups are typically prevented. There was no structural evidence that polymorphic transitions were predicted within this data set using the All Atoms minimization method.

14. Perhaps even more troubling, such behavior suggests that the global minimum for such a calculation does not correspond to any of the known structures. Although potentially an indicator of a yet undiscovered more stable form, this is more likely a reflection of inadequacy in the computational method.

15. Dunitz JD. 2003. Are crystal structures predictable? Chem Comm 2003:545-548.

16. Mooij WTM, van Eijck BP, Kroon J. 2000. Ab initio crystal structure predictions for flexible hydrogenbonded molecules. J Am Chem Soc 122:35003505 .
17. Vodak DT, Kim K, Iordanidis L, Rasmussen PG, Matzger AJ, Yaghi OM. 2003. Computation of aromatic $\mathrm{C}_{3} \mathrm{~N}_{4}$ networks and synthesis of the molecular precursor $\mathrm{N}\left(\mathrm{C}_{3} \mathrm{~N}_{3}\right)_{3} \mathrm{Cl}_{6}$. Chem Eur J 9:41974201.

18. Compass force field was used for sulfathiazole, sulfamethoxazole, and indomethacin. Dreiding force field was used for acetaminophen and nabumetone. In all cases, high convergence criteria were used.

19. Segall MD, Lindan PJD, Probert MJ, Pickard CJ, Hasnip PJ, Clark SJ, Payne MC. 2002. First-principles simulation: Ideas, illustrations and the CASTEP code. J Phys: Condens Matter 14:27172744.

20. Perdew JP, Burke K, Ernzerhof M. 1996. Generalized gradient approximation made simple. Phys Rev Lett 77:3865-3868.

21. (a) Boldyreva EV, Drebushchak VA, Paukov IE, Kovalevskaya YA, Drebushchak TN, 2002. DSC and adiabatic calorimetry study of the polymorphs of paracetamol-an old problem revisited. J Therm Anal Calorim 77:607-623; (b) Urakami K, Shono Y, Higashi A, Umemoto K, Godo M, 2002. A novel method for estimation of transition temperature for polymorphic pairs in pharmaceuticals using heat of solution and solubility data. Chem Pharm Bull 50:263-267; (c) Price CP, Grzesiak AL, Lang M, Matzger AJ, 2002. Polymorphism of nabumetone. Cryst Growth Des 2:501-503; (d) Giron D, 1995. Thermal analysis and calorimetric methods in the characterization of polymorphs and solvates. Thermochim Acta 248:1-59. 